\title{
STUDY ON THE QUALITY CONTROL METHODS OF CLUSTER-BASED REMOTE SENSING IMAGE PROCESSING
}

\author{
L. Xia, L.B. Zhao, X.P. Zhang, X.M. Zhou \\ The First Institute of Photogrammetry and Remotes Sensing, Xi’an, China \\ accelerate_1@163.com
}

KEY WORDS: cluster, quality control, aerial triangulation, DOM

\begin{abstract}
:
Along with the advances in technology, modern surveying technology developed rapidly, a series of high-tech equipments have been invited and developed. A lot of cluster-based Remote Sensing image processing systems, at home or abroad, have been produced and used in production, such as PixelFactory、PixelGrid、CIPS etc. Been popularized and used, processing technology and procedure have changed to be different from the old pattern, and traditional ways of quality control no longer meet the need of new technology. Above all, it is of vital importance for us to find new ways of procedure quality control for the geographical spatial data quality certification under new technology system. Based on the mainstream image fast processing systems - PixelFactory and PixelGrid, the nodes and methods of quality control during image processing will be studied in this paper, including image preprocessing AT、DSM、DEM、ortho-rectification、mosaic etc. After that, the feasibility of quality control ways proposed will be verified by actual production project. All the advices can be used as references for large-scale application of new technology in information surveying system.
\end{abstract}

\section{INTRODUCTION}

Being developing for several years, our surveying and mapping technology had come to the digital field, and now heading to information surveying. Though the nature of information surveying system is still digitalization, which is more fitting to the need of modern social and technology development. For instance, the fast acquisition and update of geographic spatial information, intelligent disposing and integration, large scale production and webified distribution, each of which is an important part of fusion of geographic spatial information resources, and providing multi-scale ,multi-temporal , multiform services .

Modern remote sensing technology is of vital importance in fast acquisition of geographic spatial information. As the technology of remote sensing been developed, ways of global observation and different kinds of sensors have been found and put into use, which lead to the explosion of quantity and increasing computation of remote sensing data, meanwhile, the development of society calls for higher quality and faster processing. Apparently, traditional way of image production is not able to meet the need of clients, so in order to process such a huge quantity of data, cluster processing system has been introduced into remote sensing data production, which is improved to completely fit the character of remote sensing data and support multi-level parallel processing, and is the inexorable trend of high-performance remote sensing data processing technology.

\section{INTRODUCTION OF REMOTE SENSING DATA CLUSTER PROCESSING SYSTEM}

Generally speaking, cluster processing technology is to divide one mission into several sub-missions that can be operated separately on the cluster processing platform, in which, each of the computer nodes is equipped with same hardware and software. Regarding to remote sensing data processing, standardization slicing of the data is the base of mission disintegration and parallel processing. Remote sensing data can be processed fast and efficiently with the parallel processing system and the operation capability can be enhanced by enlarging the scale of cluster.

So as to put remote sensing data clustered processing into reality, there must be 3 conditions to be satisfied: (1)cluster. A cluster must contains at least 2 processors, which are connected by network and can be communicated; (2)Remote sensing data can be processed paralleled. The data processing can be divided into several sub-jobs, and those sub-jobs can be carried out simultaneously. (3) Parallel programming. The cluster system provided parallel programming environment, the parallel processing algorithm can be designed specifically.

Because of way of remote sensing data acquisition and contents of data itself, we have to choose scheme and design the procedure based on the character of remote sensing image and our purpose. From the angle of parallel processing, there are 4 characters of remote sensing data processing: mission decomposed, which supports separate management, data decomposed, function decomposed, making customs choosing their own procedures; different sizes of operation areas .

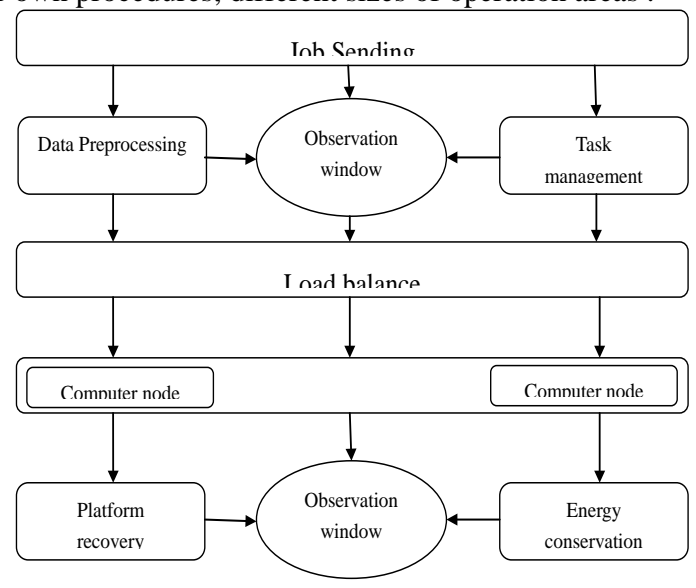


Figure 1. Frame of cluster remote sensing image processing system

With the character of comprehensively processing multiresource remote sensing data, huge data throughput, higher accuracy of algorithm, multi-mission control and management, etc., high performance remote sensing data cluster processing system made up the shortage of traditional remote sensing image production, such as low throughput and automation. So it is of vital importance for introducing cluster data processing system into production, which satisfied the large scale remote sensing data processing.

\section{CLUSTER REMOTE SENSING IMAGE PROCESSING SYSTEM PROCEDURE AND QUALITY CONTROL NODES}

Now the main stream cluster image processing systems at home and overseas, such as PixelFactory, PixelGrid, CIPS, are already been used in production. We have found the quality control procedure by practicing in different projects.

Now taking PixelFactory as an example, we will introduce the procedure and quality control nodes of cluster image processing system.

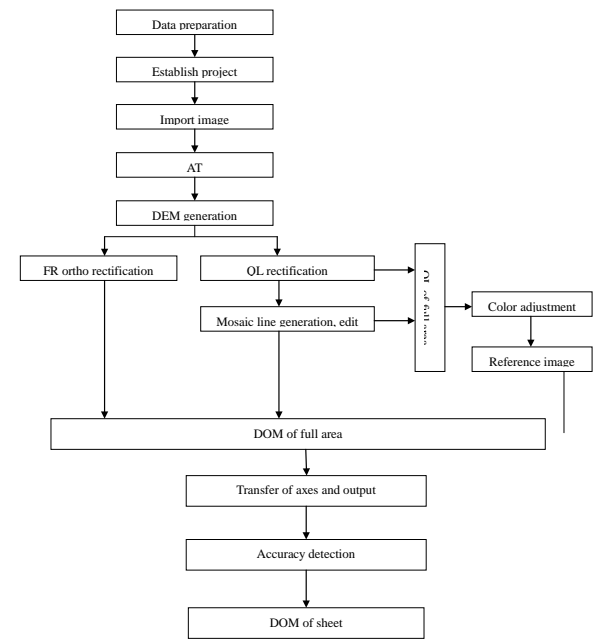

Figure 2. working flow of PixelFactory(for aerial photo) Based on the picture above, the major procedures referred in PixelFactory in cluster image processing are: data preparation、 project generation、 image import、AT、DEM production、 image ortho-rectification、 uniform radiation and color 、 image mosaic etc., And AT, DEM production and DOM production play vital roles in quality control of data processing.

\subsection{Aerial triangulation}

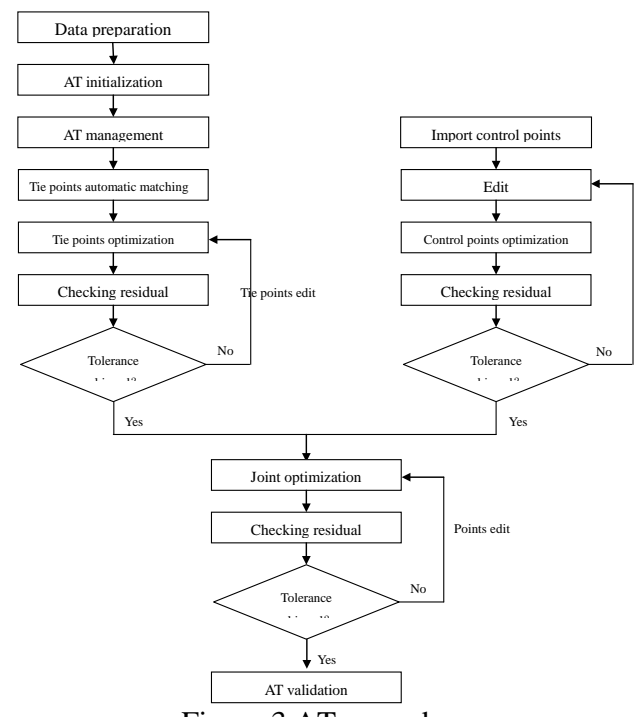

Figure.3 AT procedure

Generally, there are 3 types of optimization during aerial triangulation. First of all, it is tie points matching and optimization for free net adjustment, which is aiming to relative orientation regaining the relationship among images; second, adding control points for control net adjustment and optimization, aiming to optimize the stabbing accuracy of control points; last but not least, joint optimization for absolute orientation. Each of them is inevitable in AT. The key points of quality control during this procedure are to estimate accuracy of tie points and distribution of control points.

In cluster image processing system, tie points are matched automatically. According to different images and different acquisition quality, we should adjust the sample interval, searching window size, detection range, contrast ratio and similarity for different demand for tie points distribution of different images. If the automatic matching still cannot achieve the expected precision, we should add or delete matching points manually. By matching, edit and optimizing matching points over and over again, until the expected precision is achieved, then we can end the procedure. Under normal circumstances, the accuracy requirements of tie points are: error of image space is less than 1 pixel, average error is 0 pixel and error of mean squares is about 0.3 pixel.

Aiming at different data resource, there are 3 ways to get the control points, surveying from filed work, interpretation from $\mathrm{DEM}$ results and matching the reference DOM/DEM. Considering the accuracy, we should use surveying results from filed work while processing large-resolution aerial photos. The control points must be edited, changing to the demanded form to import into PF, which will be used by combining with the tie points and optimized. In the process, the operators must confirm the position of control points in according to the description of the surveying results strictly, checking the accuracy by vision. Generally speaking, the accuracy requirement of control points are: maximum error is 1 pixel at most, average error is 0 pixel and error of mean squares is about 0.3 pixel in image space; maximum error is less than the AT accuracy requirement, average error is 0 pixel and error of mean squares is $1 / 3 \mathrm{AT}$ accuracy requirement at most in object spaces.

In the process of aerial triangulation, optimization, checking and edit should be done circularly, each times, the optimization is finished, and the results must be checked and disposed. The 
next procedure of DSM/DOM production can be carried out while the expected accuracy is achieved.

\subsection{DEM production}

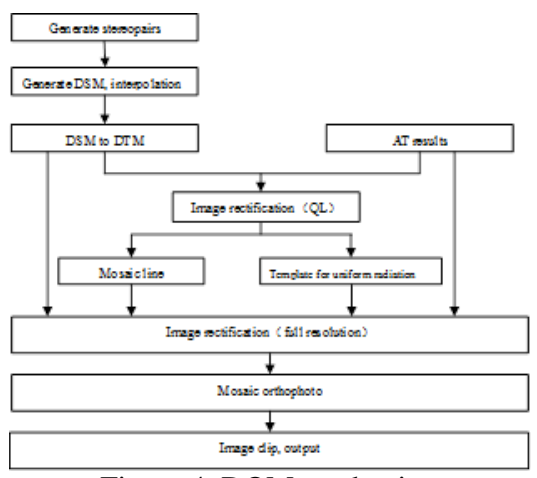

Figure 4. DOM production

The main quality control nodes of DOM production are DSM to DOM, generating mosaic line and uniform radiation and color of images.

Comparing to satellites image production, producing DOM from aerial photos needs higher resolution and accuracy DEM. Traditionally we have to get the DEM by digitalization, collecting certain feature lines and points to build TIN for further interpolation, which is time-consuming and relatively lower accuracy. Using confirmed aerial triangulation results, PixelFactory generates stereopairs between adjacent strips automatically, which can be used to build DSM and describe every details of the whole working area. It is more efficient in large area quick production.

In order to get the reality of terrain, PixelFactory provides several different algorithms to automatically edit DSM, such as high-pass filter algorithm, operators can choose different algorithm according to different land cover. Considering the accuracy of DSM, it is of vital importance for operators to choose filter algorithm and filter level. The filter results can be displayed exaggerated by 3 demotion hill-shading, which can be easily checked by vision. As mentioned above, because of understanding difference, different operators might choose different filter algorithm and level, so the proficiency of operators should be considered as a factor of influence of DSM accuracy.

Sometimes the DSM is called for as detail as possible, PF provides the function of outputting produced DSM in tiff format and editing in third-party software, such as edited by digitalization in $\mathrm{JX}-4$.

\subsection{DOM production}

After the DSM has been confirmed, it can be used in orthorectification. There are 3 parts must be prepared for orthophoto production, DSM, mosaic line and template image for uniform radiation.

In order to reduce manual work, it has introduced constraint conditions into mosaic algorithm: radiation, elevation and angle of incidence. The algorithm is designed to choose images of highest FOV, bypass the resident areas and ridges automatically. If the results are not that satisfied, it could also be edited in visualization window by manual.

Cluster image processing system has no limitation of data quantity while generating mosaic lines. Distributed parallel processing makes it possible to operate all of the images at the same times.
Using confirm mosaic lines, the quick look orthophoto which is of lower resolution, is rectified as reference image for uniform radiation. Been uniformed the radiation and color in photoshop, the QC image is used as reference histogram in the next sequence. This part also depends on the proficiency of operators. After all the preparation, the full resolution orthophoto can be rectified and clipped according to standard sheet line automatically.

During this procedure, almost all the jobs can be done by the system. What we should do to control the quality is to watch the manual work of mosaic line edit and QC image uniform radiation and color.

\section{PRODUCTION CASE}

There is an example to explain how the quality control way works during production. This is satellite image production of some area in China.

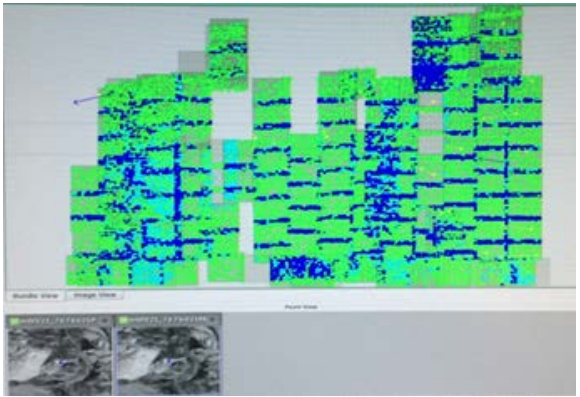

Figure 5 matching tie points automatically

Different color presents different degree of overlapping, we could delete or add tie points manually under this circumstance and optimize them over and over again until the expected accuracy is achieved.

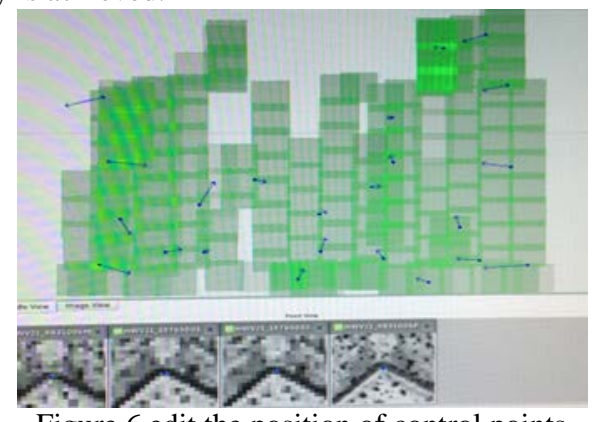

Figure 6 edit the position of control points

After importing control point and optimized with tie points, the precise position of control points can be adjusted in the windows below manually, and they must be strictly coincidence with the description with field work results.

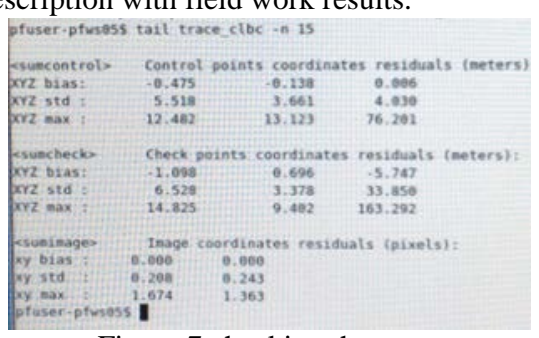

Figure 7 checking the error

Whenever the expected accuracy is achieved, finish aerial triangulation and step into DOM production. 


\section{5、CONCLUSIONS}

Comparing traditional DOM production system, there are advantages by using cluster image fast processing system: (1) more efficient through using exiting processors;(2)less control points and less field work; (3) DSM can be generated automatically but more specific, calling for less manual work; (4) all of images and elevation data could be operated intelligently during computing mosaic line;(5)comparing using single sheet image as reference histogram, QC image can restore the real radiometric and color information of the whole area; (6) batch clipping avoids non-edge- matching among DOM mapsheets.

The advantages above have changed the focusing points of quality control procedure. The results of field surveying is the guarantee for quality of control points and the inspect basis of whole production. The expected accuracy of aerial triangulation can be guaranteed by control the distribution and residual of tie points and control points, which makes sure the accuracies of relative orientation and absolute orientation no less than demanded by the custom. And the quality of DOM would be decided by filter algorithms, mosaic lines and reference image. Also the proficiency of operators plays an important role from beginning to end of orthophoto production, from surveying to final inspect. In order to control the quality of cluster image processing, all of them mentioned above should be maximized.

The flourish development of information surveying system is not only the challenge for data storage, information extraction, delivering and management, but also the challenge for way and nodes of quality control. We have to do every effort to improve the procedure of quality control for providing more reliable and timely data for Social and economic development and progress.

\section{REFERENCE}

\section{References from Journals:}

Lijun Song. 2012. Fast processing platform of remote sensing data based on improved cluster algorithm. Fujian Computer, pp.1-2.

\section{References from Other Literature:}

Yanli Shi, 2010, the architecture design and implementation of cluster-based parallel processing system for remote sensing data, chiese academy of surveying and mapping, Beijing, master dissertation.

Changqing Ren, 2010, PF Produce Handbook. 